\title{
A Research Paper: Comparison of LEACH and EEP Protocol
}

\author{
Manish Chandra \\ M.Tech Research Scholar, \\ CSE Department \\ PSIT, Kanpur
}

\author{
Sunil Kumar \\ Assistant Professor \\ CSE Department \\ PSIT Kanpur
}

\author{
Saurabh Gupta \\ Assistant Professor \\ CSE Department \\ PSIT Kanpur
}

\begin{abstract}
A mobile ad hoc network (MANET) is a collection of autonomous nodes or terminals which communicate with each other by forming a multi-hop radio network without the aid of any established infrastructure or centralized administration such as a base station. It is composed of tiny wireless sensor nodes which are mobile and dynamic nature. Due to mobility, when nodes re communicate to each other, they easily loss their energy or we can say the network life time will reduce by time. To maintain the energy efficiency of any node or network, some protocols are developed. The size of network depends on the total number of nodes in network. When size of network expands it means numbers of nodes are increases. This paper presents how life time of network can be increased by increasing number of nodes using EEP protocol. This work presents the improvement over LEACH protocol.
\end{abstract}

\section{Keywords}

Wireless Sensor Networks, LEACH, EEP, Energy.

\section{INTRODUCTION}

A WSN is composed of large number of sensor nodes which are distributed in the wireless environment. These sensor nodes can pass data to each other in different location. Due to mobile nature, the sensor nodes become passive after some time. In the wireless technology, the sensor nodes collect data, receive the information and then pass this data to other nodes or users. In wireless architecture, all nodes are mobile, so they can join or leave the network. The number of nodes can vary from network to network. In any network, the size of network can be decided by total number of sensor nodes. In any network if number of nodes is more, then the network size will be more. So to group the nodes, clustering of nodes is required so that the working of network can be done in efficient manner. If any node dies in the network then the efficiency of network will be affected. So proper clustering is required to group the nodes according to distance \& residual energy. Leach Protocol decide the next node on the basis of distance parameter. In cluster, firstly cluster head $\mathrm{CH}$ is elected on the basis of least distance from base station.

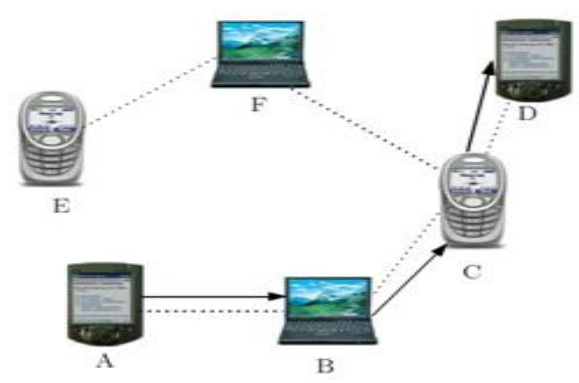

Fig 1: An Ad hoc Network Proposed Work.
Then $\mathrm{Ch}$ sends or transfers the packets to base station.In this manner, routing is done or we can say nodes are communicated in the cluster based manner. The cluster head $\mathrm{CH}$ have to take all responsibility to transfer the packet to the base station. Since the BS is typically located far away. So the nodes in a network will die very quickly. So to improve the life time of network, an improved approach has been implemented in this paper so that the efficiency of network can be increased. This work has been represented by EEP protocol. By comparison of Leach \& EEP protocol, we can easily found the improved network life time of network.

\section{PROPOSED WORK}

Leach Protocol decide the next node on the basis of distance parameter. In cluster, firstly cluster head $\mathrm{CH}$ is elected on the basis of least distance from base station. Then $\mathrm{Ch}$ sends or transfers the packets to base station. In this manner, routing is done or we can say nodes are communicated in the cluster based manner. The cluster head have to take all responsibility to transfer the packet to the base station. When cluster head become die, then another round have to take place. In large network, this procedure will become so complicated and time consuming. If number of nodes wills increases, then network size will also increases. In this type of network, so much time is consumed to perform routing. With the help of EEP protocol, the data transfer can take place easily with less time due to vice cluster head. If cluster head die, then vice cluster head can take all responsibility of cluster head $\mathrm{CH}$, so that all work can be done without any interruption. So to improve the life time of network, an improved approach has been implemented in this paper so that the efficiency of network can be increased. This work has been represented by EEP protocol [1]. Distance and residual energy are the main parameters to elect cluster head $\mathrm{CH}$ and vice cluster head. Which node has the highest energy in cluster has been selected as cluster head. This cluster head will send all data to base station BS, BS receive all the information and acknowledge the cluster head after receiving of packet. After some time, the energy of cluster head getting low or we can say the cluster head become die then vice cluster head send data packets to base station. Based on the received signal strength of the advertisement message sent by $\mathrm{CHs}$ nodes to base station, the message transfer takes place. After this Based on TDMA schedule nodes send data to their $\mathrm{CHs}$ afterwards $\mathrm{CHs}$ send the aggregated data to BS. In EEP protocol, the life time of network can be increased by increasing the number of nodes. A simple approach to perform this data gathering task is for each node to transmit its data directly to BS. But base station is located far away from nodes, so after some time the nodes die quickly due to less energy.This paper represents, the life time of network by increasing number of nodes using EEP protocol and also represent the comparison between Leach \& EEP protocol. 


\section{RESULT AND DISCUSSION}

The network size depends on the number of nodes in network. If size of network is more, then number of alive nodes should be more. The number of alive decides the lifetime of network. If number of dead nodes are more in any network, then network life time will decreases. To increase the life time of any network, various parameters are used. These parameters decide the lifetime of network. If numbers of dead nodes are more in any network, then we can say the network will be damaged very soon. So number of dead nodes will be less in nay network. I have done all simulation in MATLAB. In previous paper research, I have done simulation on number of nodes 50 and 100 . This experimental result shows that comparison of LEACH \& EEP protocol. This paper shows when number of nodes wills increases then how life time of network can be increases. This experimental result will shows that the life time of network is enhanced because of dynamic clustering and increased packet transmission rate. In this paper, I have taken only one scenario, Number of nodes $=150$. The simulation tool is MATLAB. MATLAB is a highperformance language for technical computing. These three parameters will decide the network life time i.e. alive nodes, dead nodes and packet transmission rate.

Parameters which are used for simulation in scenario two:

$$
\begin{aligned}
& n=150 \\
& P=0.1 ; \\
& E o=0.6 ;
\end{aligned}
$$

It means total number of nodes are 150 . Here $\mathrm{n}$ is number of nodes, $\mathrm{p}$ is the probability factor, Eo is the thresh hold energy value.

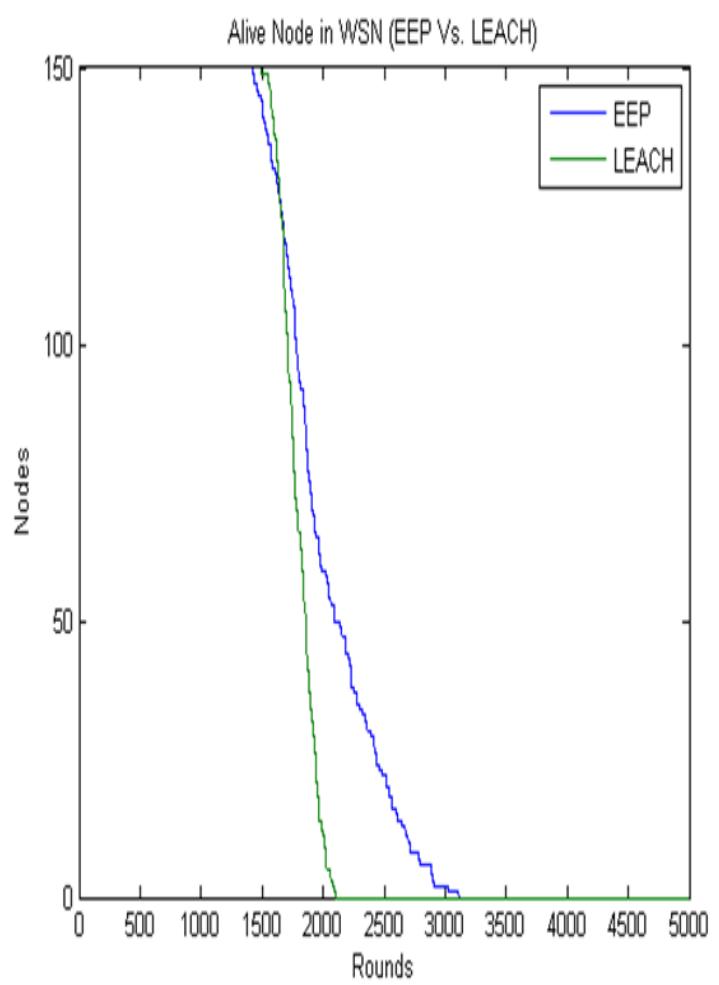

Fig 3.1 No. of Alive nodes in 150 nodes network

This graph represents the no. of alive nodes. The life of nodes in EEP is more than the LEACH protocol. The number of nodes is alive between rounds 1500 to 2000 in Leach protocol. The number of nodes is alive between 1450 to 3100 rounds in EEP protocol. So, the life of EEP is more than as compared to Leach protocol due to more energy. More nodes are alive in EEP protocol. In EEP when number of nodes is more or we can say size of network is larger, the nodes start to die early as compared to a smaller network. Due to more cluster head and vice cluster head selection, the more time and energy is consumed in EEP as compared to Leach protocol.

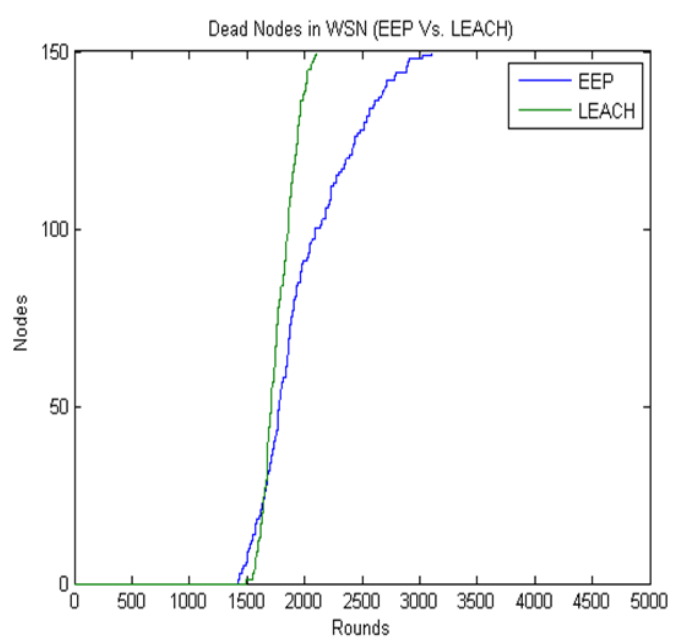

Fig 3.2 No. of Dead nodes in 150 nodes network

This graph will represent no. of dead nodes in WSN. The life of nodes in EEP is more than the LEACH protocol. The nodes start to die around round 1500 in Leach protocol. Up to 2100 round, the leach protocol completely vanished. The network life of smaller network is highly increased in EEP due the reason that time and energy is saved in cluster head selection as we have all ready a vice cluster head. So, the life of network in EEP will be more than LEACH. In EEP the nodes are alive between 1450 to 3100 rounds. When cluster head dies, the vice cluster head transfer the packets too base station. If network size increases, then more energy is consumed to select the cluster head and vice cluster head. More nodes are alive in EEP protocol. In EEP when number of nodes is more or we can say size of network is larger, the nodes start to die early as compared to a smaller network. But the life time of EEP is better than Leach protocol.

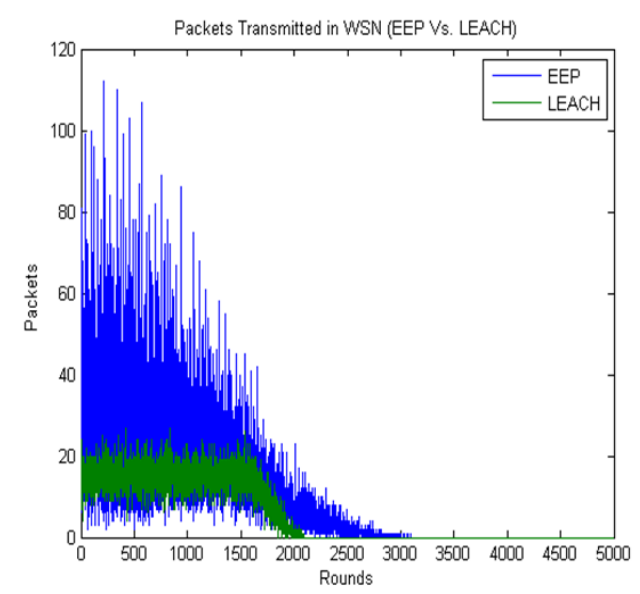

Fig 3.3 No. of Packets transmitted in 150 Nodes WSN

The number of packets transmitted to base station in EEP is higher than the LEACH protocol. Due to higher network life 
of EEP, the packet transmission rate of EEP is better than as compared to Leach protocol. In EEP the transmission is also for a longer duration i.e. up to 3100 rounds.

\section{CONCLUSION \& FUTURE SCOPE}

The main objective of this paper is to collect the information by sensor nodes. This paper present EEP protocol is more energy efficient as compared to Leach protocol. This paper represents the larger size of network with 150 nodes. The experimental result shows that the number of alive nodes is more in EEP protocol as compared to Leach protocol and also represents less number of dead nodes. It shows how the lifetime of network can be increased by increasing the network size.

In future, to increase the life time of network, we can increase the number of nodes or we can develop another energy efficient protocol with cluster head $\mathrm{CH}$, vice cluster head and another extra vice cluster head. Another parameter we can use security so that routing can be takes place without any packet loss.

\section{REFERENCES}

[1] Yingchi Mao, Zhen Liu, Lili Zhang, Xiaofang Li, "An Effective Data Gathering Scheme in Heterogeneous Energy WSNs", International Conference on Computational Science and Engineering,2009.

[2] Kun Zhang and Cong Wang, "A Secure Routing Protocol for Cluster-Based Wireless Sensor Networks Using Group Key Management ", IEEE Xplore,vol.37, pp. 178185, jan.-2008.
[3] Bolian Yin, Hongchi Shi, and Yi Shang,"Analysis of Energy Consumption in Clustered Wireless Sensor Networks" IEEE Transactions on Mobile Computing, vol. 3, pp. 272-285, July 2007.

[4] Zhao Yulan and Jiang Chunfeng,"Research about Improvement of LEACH Protocol", An Energy Efficient Protocol for Wireless Sensor Network". Application Research of Computers, No.2 pp: 209-211, Jan. 2005.

[5] Stephan Olariu and Qingwen Xu, "Information Assurance In Wireless Sensor Networks", IEEE Transactions on Mobile Computing, 3(4), pp. 317-331, Dec.2004.

[6] Zenon Chaczko, Ryszard Klempous and Jan Nikodem," Methods of Sensors Localization in Wireless Sensor Network", IEEE Transactions On Mobile Computing, VOL. 3, pp. 3, Jul-Sep 2004.

[7] Adrian Perrig , John Stankovic and David Wagner," Security in wireless sensor network",. Vol. 47, pp.6-9 ,June 2004. [8] Jan Steffan ,Ludger Fiege, Mariano Cilia and Alejandro Buchmann, "Scoping in Wireless Sensor Networks", IEEE Network, 18(1), pp.15-21, May 2004.

[8] Rishu Gupta1, Tripti Malhotra, “ an Energy Efficient Protocol To Increase Network Life In WSN “ IOSR Journal of Electronics and Communication Engineering (IOSR-JECE) e-ISSN: 2278-2834,p- ISSN: 2278-8735. Volume 6, Issue 4(May. - Jun. 2013), PP 75-81 www.iosrjournals.org 